Results 927 household-heads were recruited (including 10 seeds). Full and small RDS-samples were largely representative of the totalpopulation for most variables, but under-represented men who were younger, of higher socioeconomic status, and with unknown sexual activity and HIV status. RDS statistical inference methods failed to reduce these biases. Only 31-37\% (depending on method and sample size) of RDS-estimates were closer to the true population proportions than the RDS-sample proportions. Only $50-74 \%$ of RDS bootstrap 95\% CIs included the population proportion.

Conclusions RDS produced a generally representative sample of this well-connected non-hidden population. However, current RDS inference methods failed to reduce bias when it occurred. Whether RDS can collect the data required to reliably remove bias and measure precision during analysis is unresolved. As such, although RDS may be a feasible and cost-effective method for sampling hidden or hard-to-reach populations, RDS should still be regarded as a (potentially superior) form of convenience sample, and caution is required when interpreting findings from RDS studies.

\section{S13.4 USE OF RESPONDENT-DRIVEN SAMPLING FOR MONITORING HIV BEHAVIOURS AMONG INJECTING DRUG USERS IN THE UNITED STATES}

doi:10.1136/sextrans-2011-050102.55

A Lansky, E A DiNenno, C Wejnert. Centre for Disease Control and Prevention, Atlanta, Georgia, USA

Background Approximately 1.1 million persons in the United States are living with HIV and for $18.5 \%$ their infections are attributable to injection drug use. In 2009 there were an estimated 5063 new HIV diagnoses attributed to injection drug use. In 2002, CDC developed the National HIV Behavioural Surveillance System (NHBS) to help state and local health departments in areas with high AIDS prevalence monitor behaviours and use of prevention services in groups at highest risk for HIV infection, including injection drug users (IDU). NHBS uses a sampling method most appropriate for each group; respondent-driven sampling (RDS) was chosen as the method for NHBS-IDU. We describe implementation and key monitoring indicators from the first two rounds of NHBS-IDU.

Methods NHBS-IDU is implemented in more than 20 cities every 3 years using a standardised protocol for conducting surveys and HIV testing among persons who had injected drugs within the 12 months prior to interview. Data are analysed for each city independently and then aggregated and weighted to form national estimates.

Results During the first IDU cycle (NHBS-IDU1, conducted 2005-2006), a total of 13519 persons in 23 cities were recruited to participate, which resulted in 11471 persons included in the final dataset. A total of 10901 persons received 34038 coupons to recruit others; 13115 (62\%) coupons were returned (range by city: $52.2 \%-75.3 \%$ ). Challenges to the underlying assumptions of RDS included a somewhat high (5\%) proportion of participants who reported their recruiter was "a stranger" and limited geographic cross-recruitment, suggesting IDU networks were not linked. These issues were found for some cities and were addressed in the operational guidance for NHBS-IDU2, conducted during 2009. Data from NHBS are used to monitor national progress in HIV prevention for IDU; in NHBS-IDU1, an estimated $32.8 \%$ of IDU shared syringes, and $63.4 \%$ had unprotected vaginal sex; $66.3 \%$ had been tested for HIV, and $29.7 \%$ had participated in an HIV behavioural intervention.

Conclusions Use of RDS for NHBS-IDU has identified challenges in implementation and analysis that continue to further the development of this method for conducting behavioural surveillance among IDU in order to characterise the HIV epidemic in the USA.

\section{S14 Research in progress: updates from American STD association developmental award recipients

S14.1 GENITAL AND ORAL HUMAN PAPILLOMAVIRUS IN
ADOLESCENT MALES

doi:10.1136/sextrans-2011-050102.56

B A Weaver, D Brown, J D Fortenberry. Indiana University School of Medicine, Indianapolis, USA

Background Human papillomavirus is a common STI which causes genital warts and cancers in males. Studies of HPV in adult males are underway, but the epidemiology and natural history of HPV in adolescent males has not been investigated. The purpose of this study is to describe genital and oral HPV infections in young men. Methods A subset of young men was recruited from an ongoing study of the male adolescent penile and urethral microbiome and sexual behaviours. Participant consent and parental permission were obtained. This study was approved by the Institutional Review Board at Indiana University. Approximately every 3 months, genital swabs and an oral rinse sample were collected from each participant. Genital swabs were collected using saline wetted cotton swabs rubbed over the entire skin surface of the participant's glans penis, penile shaft, and scrotum. Participants provided oral samples by a swish and spit method using $15 \mathrm{ml}$ of mouthwash. Samples were tested for HPV using the 37 HPV type Linear Array HPV Genotyping Test (Roche). Participants completed daily cell phone diary entries and quarterly surveys about their sexual behaviours. Descriptive statistical analysis was performed using SPSS.

Results A total of 34 adolescent males were recruited, ages 14-18 at enrolment (mean 15.8; SD 1.18). The racial/ethnic identity of participants was: $19(55.9 \%)$ black, 12 (35.3\%) white, and 3(8.8\%) other. At the time of their first HPV sample collection, 19 participants $(55.9 \%)$ reported ever having vaginal sex, 8 participants (23.5\%) reported ever giving oral sex, 16 participants $(47.1 \%)$ reported ever receiving oral sex, and $5(14.7 \%)$ reported ever having anal sex. In their enrolment genital samples, HPV (high risk (HR) plus low risk (LR)) was detected in 13 of 34 participants (38.2\%); HR types were detected in nine participants and LR types were detected in eight participants. Seven participants had $>1$ HPV type. HPV was detected in both sexually active participants and those denying prior sexual contact. One participant had trichomonas at enrolment; no participants had gonorrhoea or chlamydia. In the enrolment oral samples, HPV was detected in one participant (HPV 6).

Conclusions This study provides the first information about HPV genital and oral infections in adolescent males. Genital HPV can be detected in adolescent males, and is not predicted by prior vaginal or oral sex. Oral HPV was infrequently detected in this small sample.

\section{S14.2 A STUDY OF AFRICAN AMERICAN AND LATINA WOMEN AND HUMAN PAPILLOMAVIRUS: LESSONS LEARNT}

doi:10.1136/sextrans-2011-050102.57

${ }^{1,2} \mathrm{~L}$ Bonney, ${ }^{3} \mathrm{M}$ Fost, ${ }^{1} \mathrm{Y} F$ Wang, ${ }^{1} \mathrm{~V} \mathrm{~L}$ Green, ${ }^{2} \mathrm{G}$ Wingood, ${ }^{1,2} \mathrm{C}$ del Rio, ${ }^{3} \mathrm{R}$ Rothenberg. ${ }^{1}$ Emory University School of Medicine; ${ }^{2}$ Emory University Rollins School of Public Health; ${ }^{3}$ Georgia State University

Background African American and Latina women in the United States suffer from sexually transmitted infections at higher rates than white women. It is particularly important to prevent HPV in these groups as they also suffer disproportionately from cervical cancer. From 2006, a prophylactic HPV vaccine has been approved for use in girls and women aged 9-26 years. However, public health focus has been on young girls and teens. There are limited options 